\title{
Multi-level Protection Building for Virus Protection Infrastructure
}

\author{
Si-Choon Noh ${ }^{1}$, Dong Chun Lee ${ }^{2}$, and Kuinam J. Kim ${ }^{1}$ \\ 1 Dept. of Information Security, Kyonggi Univ., Korea \\ nsc1@kt.co.kr \\ 2 Dept. Computer Science, Howon Univ., Korea
}

\begin{abstract}
This paper proposes an improved multi-level virus protection infrastructure as a measure for correcting these weaknesses. Improved virus protection infrastructure filters unnecessary mail at the gateway stage to reduce the load on Server. As a numerical result, number of transmission accumulation decreases due to the reduction in the CPU load on the virus wall and increase in virus treatment rate.
\end{abstract}

\section{Introduction}

Computer virus is becoming increasingly sophisticated on a technical level. The base of the recommended virus protection strategy dictates that changed protection infrastructure is required to effectively address changed attack pattern [5]. Virus infection is infiltrated to local drive through floppy drive, email, Internet downloads, and various types of macro-enabled application. Moreover, HTTP based Web traffic, FTP based file transmission, and synchronized PDA data are received to this place [1. Application of scanning in letters, stored in mail box, is difficult, and virus infection takes place in any case whether forwarding, opening, replying or using file 4. Malignant code that infiltrated the target system through all types of routes begins to act as the same time when the user activates the operation. Proliferated worm virus continues to infect the inside while increasing the outbound traffic dramatically, which in turn increases session on the gateway level[2], [3].

The dualized protection method that is divided into server and PC, which does not have the function to block the virus that circulates in the network since it restricts the protection zone to the Server and PC. Characteristics of network traffic differs by the type of TCP/IP service, and single method based treatment by using one vaccine does not effectively block diverse infiltrations.

\section{Multi-level Virus Protection Infrastructure}

Multi-level virus infrastructure is applied on the network infrastructure, traffic route, protection zone, gateway area protection method, server protection method, anti-virus software configuration. 
Protection Infrastructure Re-configuration: Gateway area and internal network virus wall area for the Web and email traffic filtering are added on to the structure, layered into three stages, from the exit point to the firewall, server and client areas to configure the defense layer consisting of five stages. The reason for SMPT gateway installation is that email protection gateway is re-set as the representative route for the influx of many emails in order to block virus before entering the intranet. Virus wall at the internal network is the function that blocks the internal circulation of the virus that is already infiltrated, and sets the new protection layer.

Traffic Route Re-setting: Network Traffic is divided into two channels by the type of channel, and the protection infra structure is made into stages accordingly. The traffic routes that are classified are internal intranet area and DMZ area. The internal intranet area ranges from the external contact point to the end user, and it is Exterior Router $->$ Web switch $->$ Firewall $->$ Web switch $->$ Interior Router $->$ Servers $->$ Client. DMZ area ranges from the external contact point to all types of server areas that are accommodated within the DMZ, and it is Exterior Router $->$ Web switch $->$ Firewall $->$ Web switch $->$ DMZ $->$ Servers. Re-set traffic route is normal traffic circulation route and it is also virus infiltration route.

Execution of Gateway Level Protection: There are mail gate, and web gate / internet proxy at gateway level. In order to protect data from virus infection, it is necessary to execute before virus reaches to the core information on the network, and it should be executed, targeting Web traffic and SMTP traffic. Virus filtering checks whether infected with virus or not at the packet unit level, and deletes if found whereas contents filtering is the function that blocks when a specific keyword is found in the email title and main body. Email filtering is the function that restricts the permitted size of email. And file filtering is the function that blocks by checking specifically attached file name or extension in advance. Spam filtering is the function that blocks the mail that is dispatched continuously [5]. SMTP Scanner executes scanning at a point where incoming, outgoing, email and attachment pass through email gateway. It protects file by mobilizing server based solution at the SMTP email server unit.

Execution of Virus Protection Against Internal Network Circulation: Vaccine for client blocks virus in PC, but it cannot inspect the mail's attachment file that is latent in server. When server's DB is infected, immensely adverse results face the overall network. Compared to many individual client protections that are placed widely, server unit protection is more effective and powerful. Blocking virus that circulates in the intranet requires separate installation of virus wall system at the foregoing part of the local file server or scanning with software embedded method on each server.

Virus Protection Zone Expansion: Shared folder is a form of database that is provided by the groupware exchange server to enable network user to share information and data. Groupware protection should enable real-time inspection of shared folder. Groupware (MS Exchange) provides NNTP to ensure relatively 
easy use of newsgroup, and real-time virus protection should be made possible for virus infection file when it comes to newsgroup.

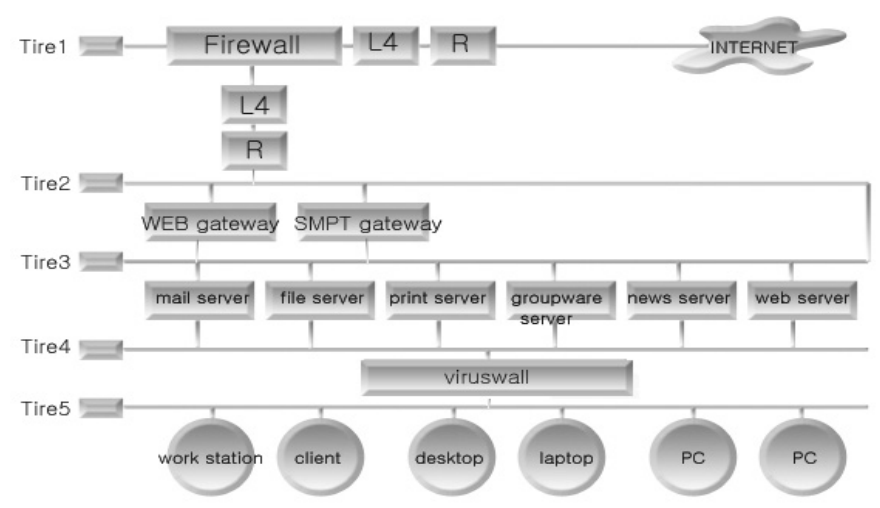

Fig. 1. Improved Protection Infrastructure

Fig.1 shows improved protection infrastructure. Infrastructure is transformed into five-stage blocking from two or three-stage blocking. And filtering and protection are executed for the entire server at the gateway level, and internet traffic route ensures differentiated protection by dividing into three, that is, SMTP, Web, and other traffic. Protection against virus that circulates in the network is conducted by collocating virus wall at the entry/exit point of local file server, and real-time protection network is configured for the client level, the infrastructure of gateway.

\section{Performance Analysis}

Gateway protection and virus blocking performance on the mail system are selected for measurement. Virus wall system for testing, used in this measurement, is E3500 system, and the software used is the virus wall of the Secureworks. Total throughput time increased to $2 \sim 3 \%$ level after the installation of the Gateway virus wall. All types of powerful filtering functions filtered $30 \sim 40 \%$ unnecessary mail among the total number of mails. When Sobig virus spills out 3,000 mails per hours, gateway Virus wall filters them first. Influx of malignant code is blocked by executing virus inspection by transmitted influx mail at the gateway first, and the mail, filtered once, is searched again by attachment file name, title, main body, and form of filtering to block abnormal mail from the network transmission process. Instead, it is stored for a specific period of time to enable re-dispatch of false positive mail that is normal mail classified as spam is enabled. Mail virus wall CPU load increased momentarily up to a maximum of $100 \%$ due 
to virus, which causes process processing delay and email transmission delay, but virus wall load stabilized at fewer than $60 \%$ on the improved structure due to virus blocking. When virus increased drastically, $10 \%$ of contaminated mail that could not be treated was transmitted to mail server, but this figure decreased to $3 \%$ after structure improvement. During the aggravation of virus wall load, the number of mails on standby for transmission reached up to 56,000 per day, but this number decreased considerably during the reduction of virus wall load.

Table 1. Comparison of Performance Analysis

\begin{tabular}{|c|c|c|}
\hline Catciory & Cieneral istrecture & Inzroved Srecturt \\
\hline Load of Virs wall CuP & Instant Maxims 100\% & Instam Masimum 6e \\
\hline $\begin{array}{l}\text { Namber of embels after } \\
\text { Failare of cure of virus }\end{array}$ & $10 \%$ & $3 \%$ \\
\hline $\begin{array}{c}\text { Trafnostandby on the } \\
\text { sendinaideily] }\end{array}$ & 56,000 & 500 \\
\hline $\begin{array}{l}\text { Masimum mamber of } \\
\text { Procoss on the sydem }\end{array}$ & 3000 & 10000 \\
\hline
\end{tabular}

\section{Conclusion}

This paper conducted research into improved effective model from the infrastructure configuration aspect. In order to ensure effective virus blocking, this paper emphasizes that a comprehensive approach through infrastructure improvement and combination of scanning tool is the only measure for preparing against today's environment of virus infiltration. The proposed method is a measure developed at a time when a permanent technological solution to virus is yet to be developed.

\section{References}

1. J. Hruska, "Computer Virus and Anti-virus Warfare" Ellis Horwood, 19962

2. P.Denning, "Computer under Attack Intruders, Worms, and Virus", AddisonWesley, 1998

3. F.Cohen, "A short Course on Computer Viruses". ASP Press, 1990

4. F.Cohen." Computer Viruses. PhD thesis", University of Southern California, 1996.

5. Rainer Link, "Server-based Virus-protection on Unix/Linux", University of applied science Frut wangen, 2003. 